\title{
Group Risk-Taking in a Two-Choice Situation: Replication, Extension, and a Model ${ }^{1}$
}

\author{
RoBert B. ZajonC \\ The University of Michigan
}

AND

\begin{abstract}
Robert J. Wolosin, Myrna A. Wolosin, and Steven J. Sherman Indiana University
\end{abstract}

\begin{abstract}
In an attempt to determine if individual risk preferences change under group conditions, individuals and groups were observed when making binary decisions. The expected values of the outcomes were held constant while the probabilities of the two events varied across conditions. As previously, it was found that when the probabilities of the two events are .6 and .4 , a conservative shift is obtained. When these probabilities are .8 and .2 , however, there was a tendency for groups to shift toward risk. A two-stage parameter-free model was suggested to account for individual and group choices.
\end{abstract}

Current theories which deal with the so-called "risky shift" phenomenon (e.g., Kogan and Wallach, 1967) assume that the differences between decisions made by persons alone and in groups come about because of changes in risk preferences. It is assumed by these theories that there exist psychological processes deriving from the group situation which lead the individual to change his "normal" level of risktaking. While there is widespread agreement that changes in individual risk preferences do indeed occur when the individual joins a group, there is no particular agreement about the psychological processes that are implicated in these changes. One theory maintains, for instance, that changes in the members' risk preference occur because responsibility for the decision is diffused throughout the group (Wallach, Kogan, and Bem, 1964). Another assumes that the group setting gives the individual an opportunity to learn that, relative to cultural standards, his risk level is lower than he previously imagined (Brown, 1965; Hinds, 1962; Stoner, 1968). Other theories assume that risk level changes because the person is more familiar with the decision problem in the group setting because

\footnotetext{
${ }^{1}$ We are grateful to Mr. Edward J. Lichstein, who helped us in collecting and tabulating the data.
} 
he then sees it a second time (Bateson, 1966; Flanders and Thistlethwaite, 1967), or that group members with higher risk preferences are more influential and persuade the others to choose riskier courses of action (Clausen, 1965; Wallach, Kogan, and Burt, 1968).

A recent study by Zajonc, Wolosin, Wolosin, and Sherman (1968) attempted to determine whether the risky shift phenomenon could be explained, without assuming such changes in individual risk preference. The task used in that experiment was different from the so-called "choice dilemma" task typically employed in other studies of group risk-taking. It required subjects to predict which of two events would occur on each of a series of 360 trials. One event occurred with a probability of .6 , and the other with a probability of .4 . Subjects received 1 cent for a correct anticipation of the first event and $1 \frac{11}{2}$ cents for a correct anticipation of the second event, and they suffered no losses for inaccurate predictions. For six blocks of 30 trials all subjects worked alone. Subesequently, one-half continued alone while the remaining subjects were formed into threeman groups which made the same predictions jointly.

The argument that changes in individual risk preferences are not a necessary condition of the risky shift phenomenon involved the assumption that groups use the majority rule in arriving at joint decisions. If groups proceed by majority rule, a group shift toward greater risk would necessarily occur if in our two-choice situation the average of the group members' risk preference were above .5. If, on the other hand, individual risk preferences averaged to some value below .5, conservative shifts could be expected (Zajonc et al., 1968, p. 92). These derivations about the probability that the group will choose a more risky course of action when the risk preferences of its members are known required that there be no changes in their risk preferences when they joined the group.

In contrast to most of the experimental literature in this area, the groups in our experiment shifted not toward risk but toward caution. But, contrary to what one would expect, the individual levels of risk preference were not below but above .5. Moreover, a closer analysis of the data revealed that the greater was the individual members' preference for risk, the greater was their shift toward caution when they were formed into a group.

The conclusion drawn from these results was that there probably isn't any simple decision scheme which the groups could have used and behaved as they did without a concomitant change in individual preferences for risk or for some component of risk. It was speculated, therefore, that individuals changed their choice behavior when placed in the group setting, and that this came about through changes in the values of decision outcomes. Perhaps, once in a group, individuals attached a 
greater cost to being wrong and a greater value to being right, and, consequently, refrained from suggesting or supporting the risky alternative which, of course, had a higher probability of being wrong. If the psychological value of being correct in the group situation is denoted by $x$ then the expected value of the frequent alternative became .6 cents $+.6 x$, while the expected value of the infrequent alternative became .6 cents $+.4 x$. Under these conditions a preference for the more frequent alternative was simply a matter of maximizing expected value and not of change in risk preferences. ${ }^{2}$

However, because the individual choices averaged rather close to an indifference level (.522), and because there were no other direct supporting data, the above remained merely a conjecture. The purpose of the present experiment is to determine whether these results will also obtain when individual choices are made initially more extreme and also, because of the peculiar nature of our findings, to replicate the previous study. In one condition of the present experiment the probabilities of the two events were .8 and .2 , and in the other, .6 and .4 , as before. In both conditions the expected values of the outcomes associated with each choice were the same $(.6 \phi)$. The payoffs were $\stackrel{z}{z}$ and 3 cents in the first condition, and 1 and $1^{1 / 2}$ cents in the other. If our conjecture about the results of the first study were correct, we should obtain a stronger conservative shift in the $.8-.2$ condition than in the $.6-.4$ condition. According to this conjecture, in the former condition the expected value of the more frequent alternative is .6 cents $+.8 x$ while that of the less frequent alternative is .6 cents $+.2 x$, a considerably greater difference between the expected values of the alternatives than in the $.6-.4$ condition. If there were indeed a change in the expected value of the outcomes which derive from the group situation, then a greater shift toward conservatism should be observed in the $.8-.2$ condition than in the $.6-.4$ condition.

\section{METHOD}

\section{Subjects}

Eighty-four subjects, all male undergraduates, were recruited from the paid psychology subject pool. A minimum of $\$ 1.25$ per hour was guaranteed. When

${ }^{2}$ Pruitt and Teger (1968) claim that cautious shifts occurred in our study because the groups had only 20 seconds to discuss their choices. While this is true for each trial, Pruitt and Teger ignore the fact that the groups had three minutes before each block of 30 trials, and could comfortably discuss their choices for the next 30 trials. Moreover, nothing prevented the subjects from discussing choices during the course of each trial block. 
scheduled, subjects were told neither about the purpose of the experiment nor that they could earn a greater sum of money during its course.

\section{Apparatus}

Two 1/25-watt lights, mounted on a small panel, served to signal stimulus events. One light was marked either 1 cent or 3 cents. The other was marked either $1 \frac{1 / 2}{12}$ cents or 3 cents. For half of the subjects in each condition, the left stimulus light was associated with the higher payoff, and for the remainder, with the lower payoff. A punched-tape program controlled the timing and sequence of stimulus events. Subjects were equipped with switches for making their choices and poker chips for tallying their winnings. Stimulus events and subjects' responses were recorded on an Esterline-Angus Event Recorder.

\section{Design and Procedure}

All subjects were given 360 trials in 12 blocks of 30 trials each. There were two stimulus conditions to which subjects were randomly assigned:

6-.4 Condition. The two stimuli were programmed with .6 and .4 probabilities of occurrence. The payoffs for this condition were 1 cent and 13 cents, respectively.

8-.2 Condition. The stimuli were programmed with probabilities of occurrence equal to .8 and .2 . In this condition the payoffs were $\%$ cents and 3 cents. The sequence of stimulus events was random, with the constraint that one or the other of the above probabilities would be observed in each block of 30 trials.

Upon arriving at the laboratory, subjects were met by the experimenter and taken to their individual cubicles where they all worked alone for the first 180 trials. After a short while, the following taped instructions were given over an intercom system. (The instructions reproduced here refer to the .6-.4 condition. For the $.8-.2$ condition, the words “ $y_{4}$ (3) cents" were substituted in the appropriate slots.)

In front of you, you see two lights. Every seven seconds one or the other will go on. You also see two response plates. During each 7-second interval you are to press one plate or the other to anticipate which light will come on. If you press the left plate and the left light comes on, you win 1 (11) cents. If you press the right plate and the right light comes on, you win 1 r (one) cents. If you press one plate and the opposite light comes on you win nothing. Also, if you fail to press within the 7-second interval, you win nothing.

You can keep track of your winnings by the chips which you see in front of you. If you win 1 cent by anticipating the left (right) light, place a white chip into your bank. If you win $1 \frac{1}{2}$ cents by anticipating the right (left) light, place a blue chip into your bank. Your winnings will also be kept automatically. We guarantee you a minimum of $\$ 1.25$ per hour and you get to keep whatever you win over that amount; that is, you will get $\$ 1.25$ per hour, or your winnings, whichever is more.

Stimuli were presented every 7 seconds and there was a 1-minute rest period after each trial block. Blue poker chips were always associated with the high payoff light, and white poker chips with the low payoff light.

IG Treatment. At the end of the sixth trial block subjects in the Individual-Group (IG) treatment were told that the first part of the experiment was over. The experimenter then met all three subjects and led them to a room equipped with apparatus identical to that which they had used alone. Taped instructions were again issued over the intercom: 
Again you see the lights and the plates. Now you are working as a team. This time one or the other light will go on every 20 seconds. During this interval the three of you will work together to come to a unanimous decision as to which plate to press. After you decide, one of you will press the plate. We will tell you who will begin pressing, and we will also tell you when to switch. Once again, if you press the left plate and the left light comes on, each of you wins $1\left(1 \frac{1}{2}\right)$ cent. If you press the right plate and the right light comes on, each of you wins $1 \frac{1 \%}{2}$ (one) cents. If you press one plate and the other comes on, or if you fail to press within the 20-second interval, each of you wins nothing. Either you all win or all lose on every trial. Again, you can keep track of your winnings by placing the appropriate chips in your bank.

Six blocks of 30 trials, each separated by 3-minute intervals between the blocks, were given. As announced to the subjects, there was a 20 -second intertrial interval. Each subject pressed the plates during two of the six intervals.

II Treatment. At the end of the sixth trial block subjects in the IndividualIndividual (II) treatment were told that the first part of the experiment was over. They were then taken out of their cubicles by the experimenter and allowed to relax for about 3 or 4 minutes. They were not permilted, however, to speak to one another. After the rest period, they were ushered back to their cubicles and continued as previously for six blocks of 30 trials.

After the experimental session, an explanation of the experiment was given, and the subjects were paid their wages and given their winnings. They were asked not to divulge the details of the experiment to their friends or acquaintances.

\section{RESULTS}

As previously, the results are reported in percentages of risky choices. Table 1 shows these choices for the two stimulus conditions (.6-.4 and .8-.2) and for the control (II) and experimental (IG) treatments. Figure 1 graphs them for the 12 trial blocks. An analysis of variance was performed in which the between-subject factors tested wcre conditions and treatments, and in which the only within-subject factor was trial blocks, with the first and second 180-trial sessions nested within trial blocks.

TABLE 1

Percentages of Risky Choices Made by Individuals and Groups

\begin{tabular}{lcc}
\hline Condition and treatment & $\begin{array}{c}\text { First } \\
180 \text { trials }\end{array}$ & $\begin{array}{c}\text { Second } \\
180 \text { trials }\end{array}$ \\
\hline 6-.4 Condition & & \\
II (Individual-Individual) & $42.8_{\mathrm{ef}^{\mathrm{a}}}$ & $40.9_{\mathrm{ed}}$ \\
IG (Individual-Group) & $51.6_{\mathrm{g}}$ & $\mathbf{4 6 . 6 _ { \mathrm { f } }}$ \\
8-.2 Condition & & \\
II (Individual-Individual) & $34.0_{\mathrm{ed}}$ & $28.9_{\mathrm{a}}$ \\
IG (Individual-Group) & $34.8_{\mathrm{b}}$ & $37.7_{\mathrm{bod}}$ \\
\hline
\end{tabular}

a Means having different subscripts are significantly different from each other at the .05 level. 


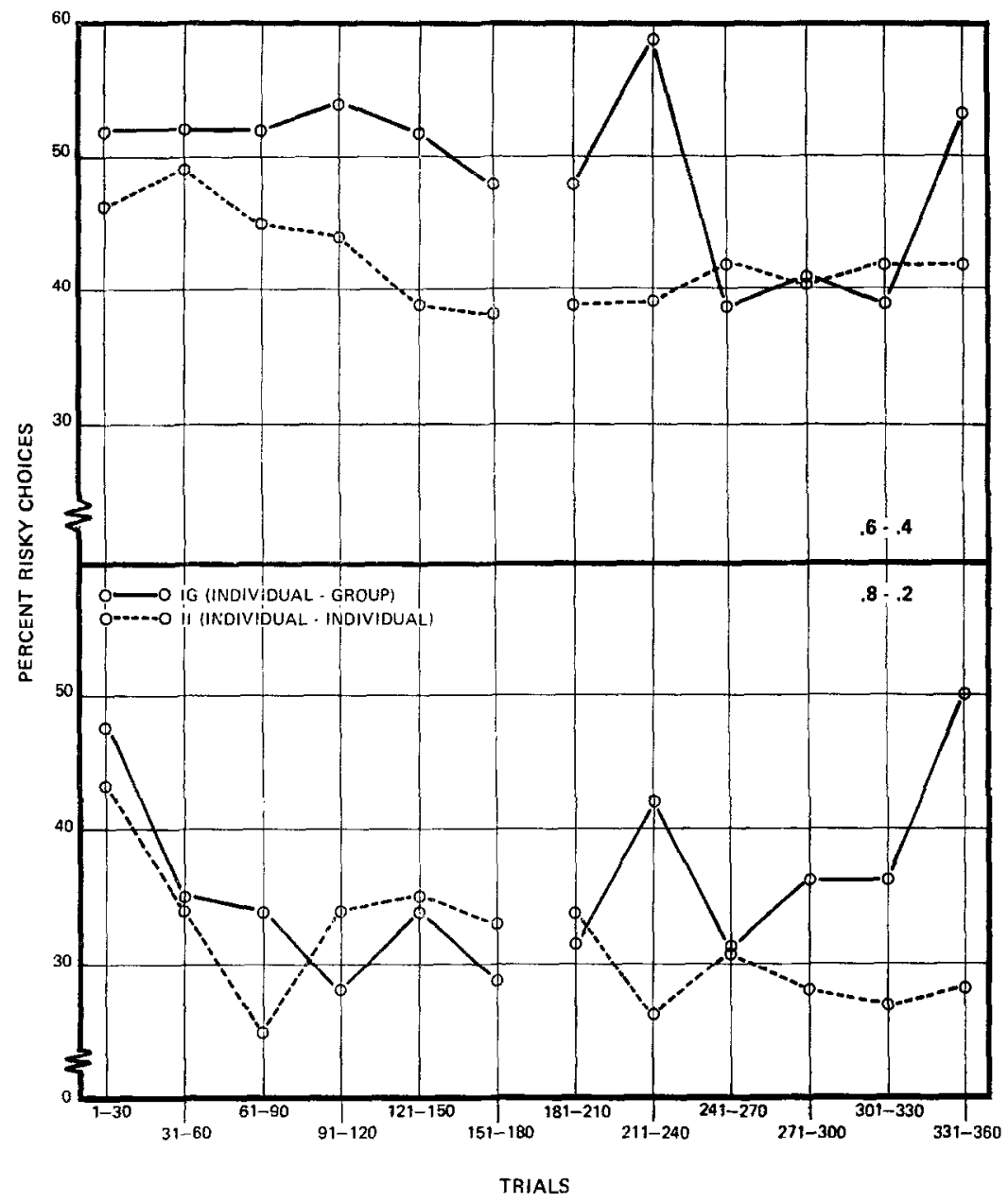

FiG. 1. Percentage of risky choices in $.6-.4$ and $.8-.2$ conditions.

The degrees of freedom in the error term were reduced because the individual scores were grouped in order to equate the reliability of the data points associated with individual and group treatments. As in the previous experiment, random "groups" of three subjects were composed from the II subjects and treated as single data points, thus achieving a comparability with the IG treatment, in which the data points are necessarily based on groups of three subjects.

This analysis of variance revealed a significant treatment effect $(F=$ 7.07, 1 and $24 d f, p<.05$ ), with the IG treatment showing a greater average number of risky choices across sessions and across conditions. There was also an overall effect of conditions $(F=27.71,1$ and $24 d f$, 
$p<.001$ ), with the $.6-.4$ condition showing a greater overall number of risky choices. But, contrary to expectations, there was also a significant interaction between treatments, conditions, and sessions $(F=3.85,1$ and $264 d f, p<.05$ ). This interaction results because the IG subjects reduced their average number of risky choices in the $.6-.4$ condition but increased it in the 8-.2 condition. Comparisons among the cell means indicate that this change is significant beyond the .05 level for the $.6-.4$ condition, and that it just fails to reach it for the $.8-.2$ condition.

As previously, a correlation was found between the prior level of risk and the amount of shift. For the .6-.4 cundition this correlation was -.49 , which is somewhat less than the -.79 found previously. However, in the $.8-2$ condition a rather substantial correlation of . -89 was found. These correlations indicate that the greater is the level of risk of the individuals working alone the greater is their shift toward caution (or lesser shift toward risk) when they are formed into three-man groups.

\section{DISCUSSION}

Two sets of findings of importance emerge from this experiment. First, the previous results were replicated. There was again a significant conservative shift in the .6-.4 condition; there was again a negative correlation between prior risk preference and the amount of shift, and also observed was the upswing in risk preference toward the close of the second session. In the present experiment, however, this upswing occurs primarily in the IG treatment. In the previous paper we speculated that this effect could be attributed to a sort of "last ditch" stance which the subjects assumed when the close of the experiment was in sight. This speculation is more cogent for the present results, however. In the present experiment, as in the previous, all subjects were scheduled for a 2-hour participation. Hence, after 10 or 11 trial blocks subjects in the IG treatment (who were given 20-second intertrial intervals in the group session, and who, therefore were in fact nearing two hours of participation at the twelfth trial block) had fairly good grounds to believe that their task was nearing the end. Subjects in the II treatment remained on a schedule of 7-second intervals throughout the experiment, and at the beginning of the twelfth trial block had worked for less than $1 \frac{1}{2}$ hours. Hence, they would be less likely to regard the twelfth trial block as the last one.

The second finding of this study is that the $.8-.2$ subjects instead of shifting toward conservatism even more than the .6-.4 subjects, changed in the direction of greater risk. Clearly, if there is a change in the individual's choice behavior when he joins the group, it cannot possibly be explained by the processes we have conjectured in the previous paper. 
The data, however, lend themselves to a somewhat different analysis, one which returns to our previous assumption that a shift toward risk or conservatism requires no changes in individual risk preferences. (This assumption, of course, does not preclude the possibility that there are conditions where shifts toward risk do occur and where there are concomitant changes in individual risk preferences of the group members.) We shall now offer a different (although still rather simple) model of individual and group risk-taking.

Since we are dealing with a form of probability learning, one of the various mathematical models for such learning could be applied to the individual data, and then extended to groups. Unfortunately, none of these models seems to fit the individual data, mainly because they have been constructed for the one-payoff case, that is, for the case where accuracy on the frequent and on the infrequent alternatives results in the same reward. The alternatives, therefore, have different expected values.

Let us then consider the following two-stage model for the case where there are two alternatives, $A$ and $B, A$ occurring with the probability $p(\mathrm{~A})$ and $\mathrm{B}$ with the probability $p(\mathrm{~B})$, and $p(\mathrm{~A}) \geq p(\mathrm{~B})$. We shall assume that under these conditions, at the onset of any trial the individual is in one of three states: (a) He is certain that A and only A will occur; (b) he is certain that B and only B will occur; (c) he believes that either $A$ or B will occur. Let us label these three states A, B, and A $\cup$ B. We will assume arbitrarily that the individual's probabilities of being in these states correspond to the objective stimulus probabilities. Thus, the probability of being in state $A, P(A)$, is equal to the probability that the event $A$ will occur times the probability that the event $B$ will not occur. For the $.6-4$ condition we have $P(\mathrm{~A})=p \mathrm{~A}(1-p \mathrm{~B})=.36$. For subjects in the same condition the probability of being in state $B$ is $P(\mathrm{~B})=p \mathrm{~B}(1-p \mathrm{~A})=.16$. And the probability of being in state $\mathrm{A} \cup \mathrm{B}$ is equal to the complement of the sum of the two above probabilities $P(\mathrm{~A})$ and $P(\mathrm{~B})$, and for the present example $p(\mathrm{~A} \cup \mathrm{B})=.48$.

We shall further assume that if the individual is in state $A$ or in state $B$ he will remain in that state and make his response accordingly for that trial. State $A \cup B$, however, is unstable, and the individual will not remain in it. If in state $A \cup B$ at the onset of the trial the individual will move either to state $A$ or to state $B$ before making a response. Again one must make an assumption about the probabilities of moving from $A \cup B$ to $A$ and to $B$, and one possibility, of course, is to assume that the movement from $A \cup B$ to $A$ and to $B$ is equiprobable. But this assumption does not correspond to the observed data. One could also assume that the movement from state $\mathrm{A} \cup \mathrm{B}$ to $\mathrm{A}$ and to $\mathrm{B}$ follows the 
objective probabilities of $\mathrm{A}$ and $\mathrm{B}$, such that in the $.6-4$ condition, for instance, on $60 \%$ of the trials the individual moves from $A \cup B$ to $A$, and on $40 \%$ to $B$. This assumption also does not fit the results. The third, equally simple assumption, is that the movement from state $A \cup B$ to $A$ and to $B$ follows the payoffs for $A$ and $B$. Thus, for instance, if A pays 1 cent and $B$ pays $1 \frac{1}{2}$ cents then the movement from $A \cup B$ to $A$ and to $\mathrm{B}$ will be distributed according to the ratio of $1: 1 \frac{11}{2}$. It is this assumption which we shall make for now. Table 2 shows the initial and terminal

TABLE 2

Initial and Terminal Probabilmies of Individual Chotces According to The Two-Stage Model

\begin{tabular}{|c|c|c|c|c|}
\hline \multirow{2}{*}{$\begin{array}{c}\text { Individual's } \\
\text { state on } \\
\text { trial } n\end{array}$} & \multicolumn{2}{|c|}{$.6-.4$ Condition } & \multicolumn{2}{|c|}{$.8-.2$ Condition } \\
\hline & $\begin{array}{c}\text { Initial } \\
\text { stage }\end{array}$ & $\begin{array}{l}\text { Terminal } \\
\text { stage }\end{array}$ & $\begin{array}{l}\text { Initial } \\
\text { stage }\end{array}$ & $\begin{array}{l}\text { Terminal } \\
\text { stage }\end{array}$ \\
\hline A & .36 & $=.552$ & & 704 \\
\hline$A \cup B$ & & .000 & & .000 \\
\hline B & .16 & 2.448 & .04 & $=.296$ \\
\hline
\end{tabular}

probabilities of being in the various states for the .6-.4 and the .8-.2 conditions. These predictions correspond fairly well to the observed results.

It should be noted that the above model has no free parameters to be estimated from the data. The predictions are made entirely from the objective stimulus probabilities, and the objective payoffs. Hence, it allows no individual variations. Yet it will be seen that for the present purposes it is fairly satisfactory. The model predicts $44.8 \%$ risky choices for the $.6-.4$ condition and $29.6 \%$ for the $.8-.2$ condition. Actually, on the last three trial blocks of the first session there were $39.7 \%$ and $51.3 \%$ risky choices in the II and IG treatments of the .6-.4 condition, and 34.9\% and $26.4 \%$ in these treatments in the $.8-.2$ conditions. The combined results average to $45.5 \%$ for the $.6-.4$ case and $30.7 \%$ for the $.8-.2$ case.

What happens when three-man groups are formed? Let us assume that the individual does not change in the group situation, and that he will vote according to the previous probabilities. Hence, before voting he will again be in one of two final stages, $A$ or $B$, with $A \cup B$ having been resolved beforehand according to the payoffs for $A$ and $B$. If this is the case then we can enumerate four initial states for each group: $\mathrm{AAA}, \mathrm{AAB}, \mathrm{ABB}$, and $\mathrm{BBB}$, where each term in the triads represents one member's individual state. If no changes in individual risk prefer- 
ences occur, and if the above model does indeed describe individual choices in the group situation, then the initial probabilities of these group states for the two conditions are as given in Table 3 below.

TABLE 3

Initial and Terminal Probabilities of Group Choices According to the Two-Stage Model

\begin{tabular}{|c|c|c|c|c|}
\hline \multirow[b]{2}{*}{$\begin{array}{l}\text { Group state } \\
\text { on trial } n\end{array}$} & \multicolumn{2}{|c|}{ 6-.4 Condition } & \multicolumn{2}{|c|}{$.8-.2$ Condition } \\
\hline & $\begin{array}{l}\text { Initial } \\
\text { stage }\end{array}$ & $\begin{array}{l}\text { Terminal } \\
\text { stage }\end{array}$ & $\begin{array}{l}\text { Initial } \\
\text { stage }\end{array}$ & $\begin{array}{l}\text { Terminal } \\
\text { stage }\end{array}$ \\
\hline $\mathrm{AAA}$ & 168 & 7.578 & & -642 \\
\hline $\mathrm{AAB}^{a}$ & $.410^{-}$ & .000 & $.440=$ & .000 \\
\hline $\mathrm{BBB}$ & .090 & $=422$ & .026 & $\Rightarrow .358$ \\
\hline $\mathrm{ABB}^{a}$ & .332 & .000 & .185 & .000 \\
\hline
\end{tabular}

a Each of these states can occur in three different ways, which are considered equivalent for the present purposes.

It is safe to assume that if the group is unanimous it will remain in that state until the termination of the trial. Groups that are in state AAA will vote $A$ and groups that are in state BBB will vote $B$. The question arises about groups in which there is disagreement. Again we shall make an assumption which takes the payoffs of the events into account rather than their probabilities of occurrence. In the .6-.4 condition a group which is in state $A B B$ has two individuals absolutely certain that $B$ will occur and that $A$ will not occur, and one individual who is absolutely certain that $A$ will occur and that $B$ will not occur. The individuals who are in state $B$ expect to win $1 \frac{112}{12}$ cents, while the person in the minority expects to win just one penny. Hence, in this group we have 3 cents against 1 cent, and it is fairly safe to assume that the two $B$ 's will convince the single $A$. In the group which is in the $A A B$ state the situation is less certain. The A's have two pennies together against the minority's 113 cents. Since the combined prize for the majority is higher, we will again say that the majority choice will prevail, and groups which are in the state $\mathrm{AAB}$ will move toward $\mathrm{A}$ with probability 1.0. These assumptions-quite tentative, to be sure-lead us to predict that in the .6-.4 condition there will be a conservative shift, and that these groups will average $42.2 \%$ risky choices. The actual figure, we note from Table 1 , is not too far from the predicted value, namely $46.6 \%$. It will be recalled that in the previous experiment this figure was closer yet to the prediction $(44.6 \%)$. 
Consider now the $.8-.2$ condition in which, contrary to our initial expectations, we found a shift toward risk. Again in the ABB state there will be a strong tendency for the majority to carry the group because together they have 6 cents against the minority's ${ }_{4}^{3 / 4}$ cents. However, the situation is definitely unstable in the $A A B$ case. Here both members of the majority expect to win together only $1 / 2$ cents, while the member betting on $B$ is sure that he will win 3 cents. It may, therefore, be somewhat difficult for the majority, under these circumstances, to bring the minority into accord. Homans' (1961) theory of social exchange holds that influencc can be exerted on the individual if he suffers no significant losses in yielding to it. He is able to resist influence, however, if such resistance promises some gains. Camilleri and Berger (1967) have recently proposed a model of group decision-making based on Homans? formulation. Their model leads to the consequence that when the expected gains of the alternatives are equal, the alternatives will be chosen with equal probability. Thus, because our data showed clearly unequal choices, even though the expected values of the two alternatives were equal, the Camilleri-Berger model cannot be applied to our data unless some assumptions are made about subjective utility of risk and caution, or the like, such that the subjective expected values of the alternatives become equal.

While various assumptions can again be made for the $\mathrm{ABB}$ case, we shall assume that when such a state exists, the group will reach decisions simply by taking turns according to individual preferences. Another way of saying this is that the state $A A B$ will be distributed in the ratio of $2: 1$ in favor of $\mathrm{A}$. We have computed the terminal probabilities for the group states in the $.8-.2$ condition according to this assumption and they are also shown in Table 3 . This assumption does predict a shift toward risk, and the predicted figure is $35.8 \%$ risky choices, which compares quite favorably with the observed $37.7 \%$.

The above tentative parameter-free model may provide a basis from which a more comprehensive formulation about risk-taking in groups may emerge. It will necessarily require some empirical parameters, and their choice will depend on future results. These parameters may be suggested by the ncgative correlations between prior risk levels and shift toward risk. What form they will take, however, is not clear to us at the moment. With the small amount of data thus far collected it is neither necessary nor advisable to elaborate the model further. Moreover, a model that utilizes no data in making successful predictions about: them (Table 4 summarizes the comparisons between predicted and observed values) deserves to be entertained for at least as long as there is no contradictory empirical evidence. 
TABLE 4

Predicted and Observed Propontions of Rigky Choices

\begin{tabular}{cccccc}
\hline & \multicolumn{2}{c}{ II Treatment } & & \multicolumn{2}{c}{ IG Treatment } \\
\cline { 5 - 6 } \cline { 5 - 6 } Condition & Predicted & $\begin{array}{c}\text { Observed } \\
\text { (last three } \\
\text { trial blocks) }\end{array}$ & & Predicted & $\begin{array}{c}\text { Observed } \\
\text { (all six } \\
\text { trial blocks) }\end{array}$ \\
\hline $.6-.4$ & .448 & .455 & .422 & .466 \\
$.67-.33$ & .407 & .423 & .362 & .388 \\
$.8-.2$ & .296 & .307 & .358 & .377 \\
\hline
\end{tabular}

There is some additional support for this model from another study, just completed, which was carried out for a different purpose, and the results of which were not known at the time the model was constructed. In that study the probabilities of the events A and B were .67 and .33 and the payoffs associated with these probabilities were 1 cent and 2 cents. The model predicts that, given the above event probabilities, the individual should make $40.7 \%$ risky choices. The observed asymptotic averages (last 90 trials of the individual session) in two different conditions, each with 24 subjects, were $41.7 \%$ and $43.0 \%$.

In one of these conditions, after the initial 180 individual trials, threeman groups were formed in which the members were required to express their own preferences on each trial, in full view of each other, and in which the group choice was arbitrarily defined according to the majority rule. The members in these groups shared the group gains equally. It is possible to determine for these groups the actual proportion of each of the four possible group states, $A A A, A A B, A B B$, and BBB. The observed proportions were $.271, .341, .264$, and .124, respectively. According to the model they should have been $.208, .429, .295$, and .067. It appears, therefore, that the model underestimates the number of unanimities. It is interesting to note, however, that the observed data nevertheless do conform to the model in general: There is a shift toward conservatism to the extent predicted. The percentage of $\mathrm{B}$ choices made by the groups according to the majority rule is $38.8 \%$, a figure not far removed from the predicted $36.2 \%$ (based on the addition of the ABB and the BBB states).

The agreement between the predicted and observed data together with the underestimation of unanimities and overestimation of mixed cases suggests that there takes place a group process which enhances conformity within a given trial but which assures independence across trials. That is, the group members attempt to maintain their initial individual risk preferences while working in a group. But on each trial 
they arrange their choices so as to maximize agreement with one another. Across a series of trials, however, they vary their choices so as to average to the same proportion as that characteristic of their individual trials. This hypothesis is supported by the fact that the observed percentage of individual choices during the group session was $41.4 \%$ as compared to the $41.7 \%$ which was the average of the asymptotic choices made by these same subjects before they joined their respective groups.

That the average of the individual choices remained the same in both sessions does not mean, of course, that no subject changed his level of risk-taking after joining the group. The absolute changes in individual risk levels were, therefore, computed and they averaged to $13.2 \%$, which is significantly different from zero. We do not know, however, how much of this figure is to be attributed to group effects and how much to a simple instability of choices. The proper comparison is a control condition in which subjects made decisions alone during the first and during the last 180 trials. This condition showed an average absolute change of $9.1 \%$-smaller than the change in the IG groups but still substantial. The difference between the amount of absolute change in risk in the individual and group conditions was not significant $(t=1.50,46 d f)$. The assumption is thus supported that no dramatic changes in individual risk preference (or in subjective expected value) occur when the individual joins the group, even when the average of group choices differs from the average of individual choices.

It should perhaps be noted in closing that, in contrast to other models of choice behavior, the model presented above places a special emphasis on outcomes. We assumed, namely, that under uncertainty the individual as well as the group will act neither so as to maximize expected value nor to maximize the accuracy of prediction, but that they will tend to follow payoffs. But, while the model deviates from the typical choice behavior formulation, it is rather consistent with current trends in research on the risky shift phenomenon, for it is characteristic of the most recent work also to pay particular attention to utilities and outcomes in dealing with the problem. Thus, for instance, working with life dilemmas, Stoner (1968) proposed that group discussion engages cultural values associated with outcomcs, while Marquis and Reitz (1968), who employed a gambling situation, suggested that group discussion enhances prior expected value.

\section{REFERENCES}

Bateson, N. Familiarization, group discussion, and risk taking. Journal of Experimental Social Psychology, 1966, 2, 119-129.

Brown, R. Social psychology. New York: The Free Press, 1965. 
Camilleru, S. F., ANd Berger, J. Decision-making and social influence: A model and an experimental test. Sociometry, 1967, 30, 365-378.

Clausen, G. S. T. Risk taking in small groups. Unpublished doctoral dissertation. University of Michigan, 1965.

Flanders, J. P., AND Thistlethwarte, D. L. Effects of familiarization and group discussion upon risk taking. Journal of Personality and Social Psychology, 1967, 5, 91-97.

Hinds, W. C. Individual and group decisions in gambling situations. Unpublished master's thesis, School of Industrial Management, Massachusetts Institute of Technology, 1962.

Homans, G. C. Social behavior: Its elementary forms. New York: Harcourt, 1961.

Kogan, N., and Wallach, M. A. Risk taking as a function of the situation, the person, and the group. In New directions in psychology III. New York: Holt, 1967. Pp. 111-278.

Marquis, D. C., AND ReitZ, H. J. Uncertainty and risk taking in individual and group decisions. 1968. Submitted to Behavioral Science.

Prutt, D. G., ANd Teger, A. I. The risky shift in group betting. Journal of Experimental Social Psychology 1969, 5, 115-126.

STONen, J. A. F. Risky and cautious shifts in group decisions: The influence of widely held values. Journal of Experimental Social Psychology, 1968, 4, 442459.

Wallach, M. A., Kogan, N., AND Bem, D. J. Diffusion of responsibility and level of risk taking in groups. Journal of Abnormal and Social Psychology, 1964, 68, 263-274.

Wallach, M. A., Kogan, N., and Burt, R. B. Are risk takers more persuasive than conservatives in group discussion? Journal of Experimental Social Psychology, 1968, 4, 76-88.

Zajonc, R. B., Wolosin, R. J., Wolosin, M. A., and Sherman, S. J. Individual and group risk-taking in a two-choice situation. Journal of Experimental Social Psychology, 1968, 4, 89-106.

(Received July 22, 1968) 